\title{
BMJ Open Impact of different dietary approaches on blood pressure in hypertensive and prehypertensive patients: protocol for a systematic review and network meta- analysis
}

Lukas Schwingshackl, ${ }^{1}$ Anna Chaimani, ${ }^{2}$ Georg Hoffmann, ${ }^{3}$ Carolina Schwedhelm, ${ }^{1}$ Heiner Boeing ${ }^{1}$

To cite: Schwingshackl L, Chaimani A, Hoffmann G, et al. Impact of different dietary approaches on blood pressure in hypertensive and prehypertensive patients: protocol for a systematic review and network meta-analysis. BMJ Open 2017;7:e014736. doi:10.1136/ bmjopen-2016-014736

- Prepublication history and additional material are available. To view these files please visit the journal online (http://dx.doi.org/ 10.1136/ bmjopen-2016-014736)

Received 17 October 2016 Revised 9 February 2017 Accepted 23 February 2017

CrossMark

${ }^{1}$ Department of Epidemiology, German Institute of Human Nutrition Potsdam-Rehbruecke (DlfE), Nuthetal, Germany ${ }^{2}$ Department of Hygiene and Epidemiology, University of Ioannina School of Medicine, Medical School Campus, University of Ioannina, Ioannina, Greece

${ }^{3}$ Department of Nutritional Sciences, University of Vienna, Vienna, Austria

Correspondence to

Dr Lukas Schwingshackl;

lukas.schwingshack|@dife.de

\section{ABSTRACT}

Introduction Lifestyle modification is one of the cornerstones in the management of hypertension. According to the most recent guidelines by the American Heart Association, all patients with hypertension should adopt the following dietary advices: increased consumption of fresh fruits, vegetables, low-fat dairy products and sodium reduction. The aim of the present study is to assess the efficacy of different dietary approaches on systolic and diastolic blood pressure in patients with hypertension and high normal blood pressure in a systematic review including a pairwise and network meta-analysis of randomised trials.

Methods and analysis We conducted searches in Cochrane Central Register of Controlled Trials in the Cochrane Library, PubMed and Google Scholar until November 2016. Citations, abstracts and relevant papers were screened for eligibility by two reviewers independently. Randomised trials were included if they met the following criteria: (1) hypertension (as mean values $\geq 140 \mathrm{~mm} \mathrm{Hg}$ systolic blood pressure and/or $\geq 90 \mathrm{~mm} \mathrm{Hg}$ diastolic blood pressure) or high normal blood pressure (mean systolic blood pressure $\geq 130 \mathrm{~mm} \mathrm{Hg}$ and/ or mean diastolic blood pressure $\geq 85 \mathrm{~mm} \mathrm{Hg}$ ), (2) age $\geq 18$ years, (3) intervention diets (different type of dietary approaches, eg, dietary approach to stop hypertension diet; Mediterranean diet, vegetarian diet, palaeolithic diet, low sodium diet) either hypocaloric, isocaloric or ad libitum diets, (4) intervention period $\geq 12$ weeks. For each outcome measure of interest, random effects pairwise and network meta-analyses were performed in order to determine the pooled relative effect of each intervention relative to every other intervention in terms of the postintervention values (or change scores). Subgroup analyses were planned for hypertensive status, study length, sample size, age and sex.

Ethics and dissemination As this study is based solely on the published literature, no ethics approval was required. We published our network meta-analysis in a peer-reviewed scientific journal.

Systematic review registration PROSPERO: CRD42016049243
Strengths and limitations of this study

- The protocol addresses the important question of which dietary approach offers the most benefits in the management of elevated blood pressure.

- The present network meta-analysis has a clearly established aim, stringent inclusion criteria, state-ofthe-art methods for data collection and quantitative and qualitative synthesis.

- Limitations include variations in trial design and regimen, adherence to dietary protocols, lack of blinding across the included intervention trials and ecological fallacy.

\section{BACKGROUND}

Due to its frequent occurrence and high impact on the development of cardiovascular and kidney disease, hypertension is one of the most challenging problems adversely affecting public health worldwide. ${ }^{1}$ The prevalence of hypertension accounts for nearly $40 \%$ of people older than 25 years worldwide, and the number of patients has increased from 600 million to a billion in $2008 .^{2}$

Lifestyle modification is one of the cornerstones of the management of hypertension. According to the most recent guidelines by the American Heart Association and the European Society of Cardiology and Hypertension, all patients with hypertension should follow dietary modifications: increased consumption of fresh fruits, vegetables, low-fat dairy products and sodium reduction. ${ }^{34}$

Accumulating evidence indicates that dietary factors have a predominant role in the management of elevated blood pressure. $^{5}$ In individuals without hypertension, dietary changes reduce blood pressure and prevent hypertension, thereby lowering the risk of blood pressure-related complications. 
Epidemiological studies suggest that even slight reductions in blood pressure will reduce the risk of cardiovascular disease. $^{67}$

Whereas it is already well established that aerobic exercise is more effective in reducing blood pressure in hypertensive patients compared with resistance training, ${ }^{8}$ the question regarding the most effective dietary approach in the treatment of hypertension and high normal pressure has not been evaluated.

To our knowledge, no up-to-date systematic review and network meta-analysis has been conducted to compare different dietary modifications in the management of hypertension and high normal blood pressure. Some pairwise meta-analyses have been published comparing dietary approach to stop hypertension (DASH), combined dietary approaches ${ }^{10}$ and lower sodium intake versus usual care/control diet. ${ }^{11}$ One of the most important questions that remain to be answered is which dietary approach offers the most benefits in the management of elevated blood pressure?

Therefore, our aim is to compare the efficacy of different dietary approaches on blood pressure in patients with hypertension and high normal blood pressure in a systematic review including a pairwise and network meta-analysis of randomised trials.

\section{METHODS AND DESIGN}

The systematic review and network meta-analysis was registered in International Prospective Register of Systematic Reviews (PROSPERO) (CRD42016049243), and reported in adherence to guidelines for network meta-analysis protocols $^{12-15}$ (see online supplementary file).

\section{Eligibility criteria}

Studies were included in the meta-analysis if they met all of the criteria mentioned below.

\section{Types of studies}

Randomised trial design comparison between different dietary approaches (eg, DASH; Mediterranean diet; vegetarian diet; palaeolithic diet; low sodium diet; low fat diet; low carbohydrate diet; high protein diet; low glycaemic index/load diet) with a minimum intervention period of 3 months according to recent Cochrane Reviews on diet and cardiovascular risk. ${ }^{16}{ }^{17}$ If randomised trials were more than one different length of outcomes (eg, 12 weeks and 12 months), we included the long-term data.

\section{Types of participants}

We considered only adults with a mean age $\geq 18$ years. Hypertension was defined according to the European Society of Cardiology and European Society of Cardiology and Hypertension as mean values $\geq 140 \mathrm{~mm} \mathrm{Hg}$ systolic blood pressure and/or $\geq 90 \mathrm{~mm} \mathrm{Hg}$ diastolic blood pressure. Moreover, all patients taking antihypertensive medication were included. ${ }^{18}$

High normal blood pressure (mean systolic blood pressure $\geq 130 \mathrm{~mm} \mathrm{Hg}$ and/or mean diastolic blood pressure $\geq 85 \mathrm{~mm} \mathrm{Hg}$ ) was also defined according to the European Society of Cardiology and Hypertension and the recently published Systolic Blood Pressure Intervention Trial (SPRINT) trial. ${ }^{18} 19$ Including patients with high normal blood pressure is of major relevance, since it is part of the metabolic syndrome diagnosis criteria. ${ }^{20}$

\section{Types of interventions}

Accumulating evidence indicates that dietary factors play an important role in the treatment of elevated blood pressure. Likewise, dietary modifications decrease blood pressure $^{21}$ and reduce the risk of hypertension in people without established high blood pressure. ${ }^{22}$ Even if modest, a reduction in blood pressure can have an important impact on the health of entire populations. ${ }^{5}$ We included all intervention trials that met the above inclusion criteria and included at least one of the following intervention diets and a control group (indirect evidence) or at least two intervention diets (direct evidence).

Eligible types of dietary approaches were as follows:

- DASH: high intake of fruits \& vegetables, low-fat dairy, whole grains ${ }^{21}$

- Mediterranean dietary pattern: olive oil, vegetables, fruits, legumes, cereals, fish and a moderate intake of red wine during meals ${ }^{23-27}$

- Low carbohydrate diet $(<30 \%$ of the total energy intake from carbohydrates, high intake of animal or/and plant protein $)^{28}$

- High protein $\operatorname{diet}^{29}$ ( $\geq 25 \%$ of total energy intake from protein)

- Low fat diet $(<30 \%$ of total energy intake from fat, high in grains and cereals $)^{28} 30$

- Vegetarian diet (no meat or fish) ${ }^{31}$

- Palaeolithic diet (lean meat, fish, eggs, vegetables, fruits, berries, and nuts; dairy products, cereals, added salt, and refined fats and sugar were excluded ${ }^{32}$

- Low sodium $\operatorname{diet}^{33}$

- Low glycaemic index/load $\operatorname{diet}^{34}$

Either energy-restricted diets, isocaloric or ad libitum diets were considered.

The following types of randomised controlled trials were excluded:

- Intervention studies solely based on dietary supplements (eg, vitamin C, vitamin E, calcium, potassium, garlic, soy protein) or single foods (eg, nuts);

- Placebo used in any form of dietary supplements (eg, potassium);

- Studies with an exercise/ medication ${ }^{35}{ }^{36}$ cointervention that was not applied in all of the intervention/control groups;

- Interventions based on very low energy diets (ie, $<600 \mathrm{kcal} /$ day)

Figure 1 shows the network of possible pairwise comparisons between the eligible dietary interventions. We identified a study that combined low sodium and a low fat diet (and did not fulfil the criteria of a DASH diet), 


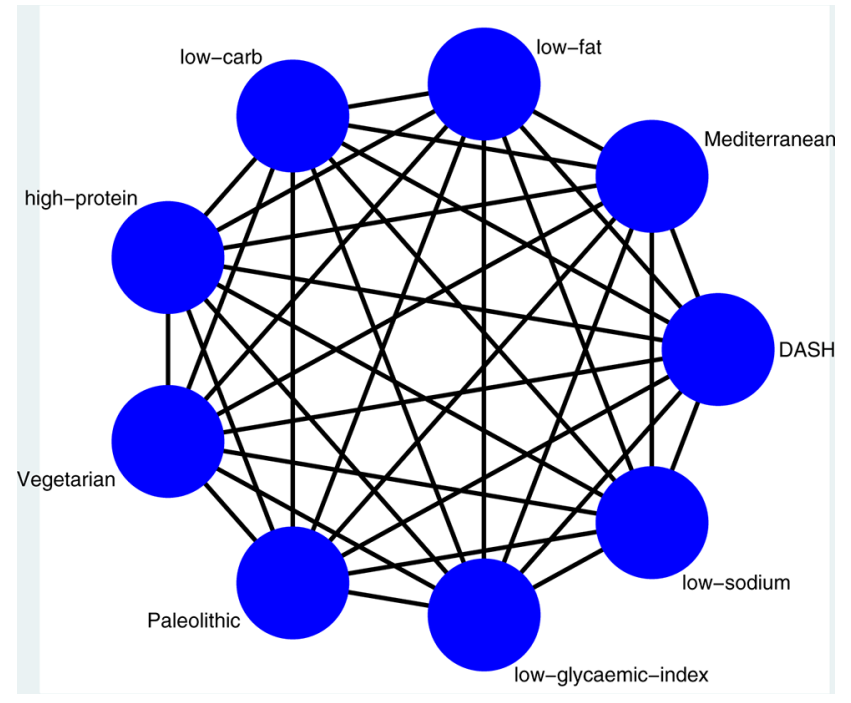

Figure 1 Network of all possible pairwise comparisons between the eligible dietary interventions. DASH, dietary approach to stop hypertension.

we handled this study as evaluating a different dietary regimen (low fat + low sodium) in the network meta-analysis. If food-based interventions also fulfilled the criteria of a nutrient-based dietary regimen, we performed sensitivity analysis for food-based versus nutrient-based dietary regimen taking into account possible overlaps.

\section{Outcome measures}

Although cardiovascular diseases are determined by variables that cannot be influenced, such as age or heritability, ${ }^{37}{ }^{38}$ there are several predictors for cardiovascular disease that can be affected by lifestyle improvements. As mentioned above, blood pressure is the most important of these modifiable risk factors. Epidemiological studies show that a reduction of approximately $3 \mathrm{~mm} \mathrm{Hg}$ in systolic blood pressure has been estimated to reduce risks of coronary heart disease by 5\%-9\%, stroke by $8 \%-14 \%$ and all-cause mortality by $4 \% .{ }^{39}$ Lowering diastolic blood pressure by $5 \mathrm{~mm} \mathrm{Hg}$ reduces the risk of stroke by $32 \%$, and ischaemic heart disease by an estimated $20 \%$. $^{40}$

Several other systematic reviews and pairwise meta-analyses have included systolic and diastolic blood pressure as outcomes. ${ }^{910}$ In order to achieve a better comparability between the data compiled by different studies, the patients should ideally hold a sitting position for 3-5 min prior to blood pressure measurement. ${ }^{18}$

\section{Search strategy}

The search was performed by LS and CS, and differences were resolved by discussion with a third reviewer (HB). We conducted searches in PubMed, Cochrane Library and Google Scholar. We searched for articles of original research by using the following search terms:

\#1 diet (MeSH Terms)

\#2 low carbohydrate OR high carbohydrate OR low fat OR high fat OR low protein OR high protein OR vegetarian OR vegan OR Mediterranean OR DASH OR dietary approaches to stop hypertension OR low glycaemic index OR low glycaemic load OR Palaeolithic OR low-calorie OR atkins OR low sodium

\#3 blood pressure OR hypertension OR diastolic OR systolic

\#4 random* NOT animals

\#5 (\#1 AND \#2 AND \#3 AND \#4)

Moreover, the reference lists from the retrieved articles, systematic reviews and meta-analyses were checked to search for further relevant studies (umbrella review of systematic reviews and meta-analyses). There were no restrictions on language or publication year. Studies published in languages other than English were translated by international scientists in our institute.

\section{Study selection process}

Titles and abstracts of all the retrieved bibliographic records were screened by two authors (LS, CS).

Potentially eligible full-text reports passing the title and abstract screening level were examined by two authors based on the a priori established inclusion and exclusion criteria. ${ }^{41}$ Discussions and consensus between the two reviewers were used to resolve disagreements by adjudication of another author. The study selection process and reasons for exclusions were outlined in a flow diagram. We included the records containing the most comprehensive information (eg, longest follow-up duration and/ or largest number of study participants), if a trial was published in duplicate.

\section{Data extraction}

The following data were extracted from each study: name of first author, publication year, country of origin, study design (randomised trial or cross-over trial), study length, number of arms, participants' sex and age (effect modifier), sample size, diagnostic criteria for hypertension, mean baseline systolic and diastolic blood pressure, mean baseline body mass index, method of blood pressure ascertainment, body weight (effect modifier), medication intake (predominately antihypertensive drugs), dietary protocols, dietary assessment method, any physical activity details, participant health status (diabetes mellitus type 2, coronary artery disease, alcohol intake, smoking), specification of the control group (if available) and where dropouts and funding source were reported.

\section{Risk of bias assessment}

Two authors assessed the methodological quality of the included trials using the risk of bias assessment tool from the Cochrane Collaboration. ${ }^{43}$ Selection bias, performance bias, attrition bias and reporting bias were assessed.

Studies were classified as being at high risk of bias if achieving fewer than four out of a maximum yield of five items at low risk of bias using the risk of bias assessment tool from the Cochrane Collaboration. Studies were classified as being at low risk of bias in general only if at least 
three out of five of the domains established a low risk of bias.

\section{Dealing with missing data}

We aimed to obtain relevant missing data from authors of the included randomised trials by mail. If the postintervention values with the corresponding SD were not available, the changed scores with the corresponding SD were imputed, according to the guidelines of the Cochrane Handbook. ${ }^{44}$

\section{EVALUATION OF SYNTHESIS ASSUMPTIONS \\ Data synthesis \\ Description of the available data}

We derived each pairwise comparison from descriptive statistics on available data and selected variables for study and population characteristics such as age, study length and outcome-relevant baseline risk factors. A network diagram was used for each outcome to present the direct comparisons between different dietary interventions and control groups. ${ }^{45}$ In these diagrams, nodes (circles) represented dietary interventions and their sizes were proportional to the sample size of each respective intervention; edges (lines) indicated direct comparisons and their thicknesses were proportional to the number of studies available. We also identified those direct comparisons having a greater influence on the network's relative effects by analysing the contribution matrix. ${ }^{45} 46$

\section{Standard pairwise meta-analyses and network meta-analyses}

To determine the pooled relative effect of each intervention (in terms of the postintervention values or the changes from baseline scores), we presented random effects pairwise and network meta-analyses. We used data on intention-to-treat analyses when available. We compared all the interventions with available direct evidence in separate pairwise meta-analyses. Heterogeneity between trial results was measured using the $\mathrm{I}^{2}$ statistic; substantial heterogeneity was considered where $\mathrm{I}^{2}$ was $>50 \%$. Study-specific effect sizes along with 95\% CIs were shown in forest plots. All available evidences were then synthesised using network meta-analysis. As extensions of the standard pairwise meta-analysis model, methods of network meta-analysis allowed for a simultaneous comparison of multiple interventions while preserving the internal randomisation of individual trials. Using a random effects network meta-analysis for each outcome, we estimated all possible pairwise relative effects and presented clinically meaningful relative ranking of the different dietary interventions. In the case of multiarm trials, we accounted for the correlation of effect sizes. Summary mean differences were presented in a league table. For each outcome, we used the distribution of the ranking probabilities and the surface under the cumulative ranking curves to estimate the relative ranking of the different diets. ${ }^{47}$ Furthermore, by assuming a common network-specific heterogeneity parameter and estimating predictive intervals, we were able to assess the impact of this heterogeneity on the relative effects with respect to additional uncertainty anticipated in future studies. ${ }^{48}$ All analyses hereby described were fitted in a frequentist framework using Stata ${ }^{49}$ (network package ${ }^{50}$ ) and our results were presented with the network graphs package. ${ }^{51}$

\section{Assumption of transitivity}

Transitivity is the fundamental assumption of indirect comparisons and network meta-analysis. Not fulfilling this assumption compromises the validity of findings from a network of studies. Changes in body weight and mean baseline age were considered as potential effect modifiers.

\section{Assessment of inconsistency}

Statistical inconsistency, meaning the presence of disagreement between the different sources of evidence, can be evaluated with help of local and global approaches. ${ }^{52}$ In our study, we used both methods. The loop-specific approach ${ }^{53}$ was used to identify loops of evidence that might present important inconsistency, and the node-splitting approach ${ }^{54}$ was used to identify comparisons for which direct estimates disagreed with indirect evidence from the entire network. Globally, a design-bytreatment interaction model and $\mathrm{I}^{2}$ statistic methods were used to identify inconsistencies jointly from all possible sources in the network. ${ }^{55} 56$

\section{Subgroup and sensitivity analyses}

In case of possible important heterogeneity or inconsistency, we explored the possible sources using subgroup and meta-regression analyses. Subgroup analyses were planned for hypertensive status, comorbidities, study length (short-term vs long-term), sample size, age and sex. Sensitivity analyses were planned for diastolic and systolic blood pressure by analysing only studies considered being at low risk of bias.

\section{Small study effects and publication bias}

We assessed the presence of small-study effects by using comparison-adjusted funnel plots. ${ }^{45}$ Contour-enhanced funnel plots ${ }^{57}$ were used to examine whether funnel plot asymmetry was likely to be explained by publication bias.

In case the publication bias was detected, we attempted to fit a selection model that represented the relationship between relative effects and probability of a study for being published and we obtained relative effects 'adjusted' for the impact of publication bias. ${ }^{58}$

\section{QUALITY OF THE EVIDENCE}

The NutriGrade tool has been especially developed for nutrition research to address specific requirements for the evaluation of meta-evidence. ${ }^{59}$ We first used this tool to evaluate and judge the meta-evidence for the pairwise comparisons. We then used our judgement about the direct comparisons and the individual contribution to the estimates within the network as described by Salanti $e t a \check{l}^{2}$ 
to draw inferences about the quality of evidence of the network meta-analysis.

\section{DISCUSSION}

According to the Global Burden of Disease Group in 2012, unhealthy diet is the leading risk factor for premature death and disability. ${ }^{60}$ Given the high prevalence and incidence of hypertension and the potential impact of diet, the conduct of the present systematic review with network meta-analysis is of high clinical and practical relevance. This network meta-analysis was one of the first to compare the direct and indirect effects of different dietary approaches in the management of hypertension and prehypertension. The results of the present network meta-analysis will influence evidencebased decision-making in treatment prescription, since it will be fundamental for reliable recommendations in the management of hypertension and prehypertension.

Contributors $\mathrm{LS}, \mathrm{AC}, \mathrm{HB}$ and $\mathrm{GH}$ contributed to the conception and design of the systematic review and meta-analysis. $\mathrm{LS}, \mathrm{AC}$ and $\mathrm{HB}$ were involved in the acquisition and analysis of the data. LS, AC, CS and HB interpreted the results. LS, $\mathrm{AC}, \mathrm{GH}, \mathrm{CS}$ and $\mathrm{HB}$ drafted this protocol. All authors provided critical revisions of the protocol and approved submission of the final manuscript.

Competing interests None declared.

Provenance and peer review Not commissioned; externally peer reviewed.

Open Access This is an Open Access article distributed in accordance with the Creative Commons Attribution Non Commercial (CC BY-NC 4.0) license, which permits others to distribute, remix, adapt, build upon this work non-commercially, and license their derivative works on different terms, provided the original work is properly cited and the use is non-commercial. See: http://creativecommons.org/ licenses/by-nc/4.0/

(C) Article author(s) (or their employer(s) unless otherwise stated in the text of the article) 2017. All rights reserved. No commercial use is permitted unless otherwise expressly granted.

\section{REFERENCES}

1. Whelton PK. Epidemiology of hypertension. Lancet 1994;344:101-6.

2. Raised blood pressure: situation and trends. Global Health Observatory. http://www.who.int/gho/ncd/risk_factors/blood_ pressure_prevalence/en/ (accessed 04 Feb 2016).

3. Smith SC, Benjamin EJ, Bonow RO, et al. AHA/ACCF Secondary Prevention and Risk Reduction therapy for patients with Coronary and Other Atherosclerotic Vascular disease: 2011 update: a guideline from the American Heart Association and American College of Cardiology Foundation. Circulation 2011;124:2458-73.

4. Eckel RH, Jakicic JM, Ard JD, et al. 2013 AHA/ACC guideline on lifestyle management to reduce cardiovascular risk: a report of the American College of Cardiology/American Heart Association Task Force on Practice guidelines. Circulation 20142014;129(25 Suppl 2):S76-99;129(25 Suppl 2):S76-99.

5. Appel LJ, Brands MW, Daniels SR, et al. Dietary approaches to prevent and treat hypertension: a scientific statement from the american Heart Association. Hypertension 2006;47:296-308.

6. Cook NR, Cohen J, Hebert PR, et al. Implications of small reductions in diastolic blood pressure for primary prevention. Arch Intern Med 1995;155:701-9.

7. Staessen JA, Li Y, Thijs L, et al. Blood pressure reduction and cardiovascular prevention: an update including the 2003-2004 secondary prevention trials. Hypertens Res 2005;28:385-407.

8. Cornelissen VA, Smart NA. Exercise training for blood pressure: a systematic review and meta-analysis. J Am Heart Assoc 2013;2:e004473.

9. Saneei P, Salehi-Abargouei A, Esmaillzadeh A, et al. Influence of Dietary Approaches to stop hypertension (DASH) diet on blood pressure: a systematic review and meta-analysis on randomized controlled trials. Nutr Metab Cardiovasc Dis 2014;24:1253-61.
10. Ndanuko RN, Tapsell LC, Charlton KE, et al. Dietary patterns and blood pressure in adults: a systematic review and Meta-Analysis of Randomized Controlled Trials. Adv Nutr 2016;7:76-89.

11. Aburto NJ, Ziolkovska A, Hooper L, et al. Effect of lower sodium intake on health: systematic review and meta-analyses. BMJ 2013;346:f1326.

12. Moher $D$, Shamseer $L$, Clarke $M$, et al. Preferred reporting items for systematic review and meta-analysis protocols (PRISMA-P) 2015 statement. Syst Rev 2015;4:1.

13. Shamseer L, Moher D, Clarke M, et al. Preferred reporting items for systematic review and meta-analysis protocols (PRISMA-P) 2015: elaboration and explanation. BMJ 2015;349:g7647.

14. Hutton B, Salanti G, Caldwell DM, et al. The PRISMA extension statement for reporting of systematic reviews incorporating network meta-analyses of health care interventions: checklist and explanations. Ann Intern Med 2015;162:777-84.

15 . Chaimani A,SalantiGhttp://cmimg.cochrane.org/sites/cmimg. cochrane.org/files/uploads/Protocol\%20for\%20Cochrane\% 20Reviews\%20with\%20Multiple\%20Interventions.pdf accessed 20 Jan 2016

16. Rees K, Dyakova M, Wilson N, et al. Dietary advice for reducing cardiovascular risk. Cochrane Database Syst Rev 2013;12:CD002128.

17. Rees K, Hartley L, Flowers N, et al. 'Mediterranean' dietary pattern for the primary prevention of cardiovascular disease. Cochrane Database Syst Rev 2013;8:CD009825.

18. Mancia G, Fagard R, Narkiewicz K, et al. 2013 ESH/ESC guidelines for the management of arterial hypertension: the Task Force for the management of arterial hypertension of the european Society of hypertension (ESH) and of the european Society of Cardiology (ESC) Eur Heart J 20132013;34:2159-219;34:2159-219.

19. Wright JT, Williamson JD, Whelton PK, et al. A randomized trial of intensive versus standard Blood-Pressure control. N Engl J Med 2015;373:2103-16.

20. Huang PL. A comprehensive definition for metabolic syndrome. Dis Model Mech 2009;2(5-6):231-7.

21. Appel LJ, Moore TJ, Obarzanek E, et al. A clinical trial of the effects of dietary patterns on blood pressure. DASH Collaborative Research Group. N Engl J Med 1997;336:1117-24.

22. Forman JP, Stampfer MJ, Curhan GC. Diet and lifestyle risk factors associated with incident hypertension in women. JAMA 2009;302:401-11.

23. Schwingshackl L, Hoffmann G. Mediterranean dietary pattern, inflammation and endothelial function: a systematic review and meta-analysis of intervention trials. Nutr Metab Cardiovasc Dis 2014;24:929-39.

24. Schwingshackl L, Hoffmann G. Adherence to mediterranean diet and risk of Cancer: a systematic review and meta-analysis of observational studies. Int J Cancer 2014;135:1884-97.

25. Schwingshackl L, Missbach B, König J, et al. Adherence to a mediterranean diet and risk of diabetes: a systematic review and meta-analysis. Public Health Nutr 2015;18:1292-9.

26. Schwingshackl L, Hoffmann G. Adherence to mediterranean diet and risk of Cancer: an updated systematic review and meta-analysis of observational studies. Cancer Med 2015;4:1933-47.

27. SchwingshackI L, Hoffmann G. Does a Mediterranean-Type Diet Reduce Cancer risk? Curr Nutr Rep 2016;5:9-17.

28. Schwingshackl L, Hoffmann G. Comparison of effects of long-term low-fat vs high-fat diets on blood lipid levels in overweight or obese patients: a systematic review and meta-analysis. J Acad Nutr Diet 2013;113:1640-61.

29. SchwingshackI L, Hoffmann G. Long-term effects of low-fat diets either low or high in protein on cardiovascular and metabolic risk factors: a systematic review and meta-analysis. Nutr $J$ 2013;12:48.

30. Schwingshackl L, Hoffmann G. Comparison of the long-term effects of high-fat v. low-fat diet consumption on cardiometabolic risk factors in subjects with abnormal glucose metabolism: a systematic review and meta-analysis. Br J Nutr 2014;111:2047-58.

31. Yokoyama Y, Nishimura K, Barnard ND, et al. Vegetarian diets and blood pressure: a meta-analysis. JAMA Intern Med 2014;174:577-87.

32. Jönsson T, Granfeldt $Y$, Ahrén B, et al. Beneficial effects of a paleolithic diet on cardiovascular risk factors in type 2 diabetes: a randomized cross-over pilot study. Cardiovasc Diabetol 2009;8:35.

33. Sacks FM, Svetkey LP, Vollmer WM, et al. Effects on blood pressure of reduced dietary sodium and the Dietary Approaches to stop hypertension (DASH) diet. DASH-Sodium Collaborative Research Group. N Engl J Med 2001;344:3-10.

34. Schwingshackl L, Hoffmann G. Long-term effects of low glycemic index/load vs. high glycemic index/load diets on parameters of obesity and obesity-associated risks: a systematic review and metaanalysis. Nutr Metab Cardiovasc Dis 2013;23:699-706. 
35. Schwingshackl L, Missbach B, Dias S, et al. Impact of different training modalities on glycaemic control and blood lipids in patients with type 2 diabetes: a systematic review and network meta-analysis. Diabetologia 2014;57:1789-97.

36. Schwingshackl L, Dias S, Strasser B, et al. Impact of different training modalities on anthropometric and metabolic characteristics in overweight/obese subjects: a systematic review and network meta-analysis. PLoS One 2013;8:e82853.

37. Kessler T, Vilne B, Schunkert $H$. The impact of genome-wide association studies on the pathophysiology and therapy of cardiovascular disease. EMBO Mol Med 2016;8:688-701.

38. North BJ, Sinclair DA. The intersection between aging and cardiovascular disease. Circ Res 2012;110:1097-108.

39. Whelton PK, He J, Appel LJ, et al. Primary prevention of hypertension: clinical and public health advisory from the National High Blood pressure education program. JAMA 2002;288:1882-8.

40. Law M, Wald N, Morris J. Lowering blood pressure to prevent myocardial infarction and stroke: a new preventive strategy. Health Technol Assess 2003;7:1-94.

41. Liberati A, Altman DG, Tetzlaff J, et al. The PRISMA statement for reporting systematic reviews and meta-analyses of studies that evaluate healthcare interventions: explanation and elaboration. $B M J$ 2009;339:b2700.

42. Moher D, Liberati A, Tetzlaff J, et al. Preferred reporting items for systematic reviews and meta-analyses: the PRISMA statement. PLoS Med 2009;6:e1000097.

43 Higgins JP, Altman DG, Gøtzsche PC, et al. The Cochrane collaboration's tool for assessing risk of bias in randomised trials. BMJ 2011;343:d5928.

44. Higgins JPT, Green S, eds. Cochrane Handbook for Systematic Reviews of Interventions Version 5.1.0 [updated March 2011, 2011. www.cochrane-handbook.org

45. Chaimani A, Higgins JP, Mavridis D, et al. Graphical tools for network meta-analysis in STATA. PLoS One 2013;8:e76654.

46. Krahn U, Binder $\mathrm{H}$, König J. A graphical tool for locating inconsistency in network meta-analyses. BMC Med Res Methodol 2013;13:35.
47. Salanti G, Ades AE, loannidis JP. Graphical methods and numerical summaries for presenting results from multiple-treatment metaanalysis: an overview and tutorial. J Clin Epidemiol 2011;64:163-71.

48. Riley RD, Higgins JP, Deeks JJ. Interpretation of random effects meta-analyses. BMJ 2011;342:d549.

49. StataCorp. Stata statistical software: release 14. College Station, TX: StataCorp LP, 20152015.

50. White IR. Network meta-analysis. Stata J 2015.

51. Chaimani S. Visualizing assumptions and results in network metaanalysis: the network graphs package. Stata $J 2015$.

52. Salanti G, Del Giovane C, Chaimani A, et al. Evaluating the quality of evidence from a network meta-analysis. PLoS One 2014;9:e99682.

53. Bucher HC, Guyatt GH, Griffith LE, et al. The results of direct and indirect treatment comparisons in meta-analysis of randomized controlled trials. J Clin Epidemiol 1997;50:683-91.

54. Dias S, Welton NJ, Caldwell DM, et al. Checking consistency in mixed treatment comparison meta-analysis. Stat Med 2010;29(78):932-44.

55. Higgins JP, Jackson D, Barrett JK, et al. Consistency and inconsistency in network meta-analysis: concepts and models for multi-arm studies. Res Synth Methods 2012;3:98-110.

56. Jackson D, Barrett JK, Rice S, et al. A design-by-treatment interaction model for network meta-analysis with random inconsistency effects. Stat Med 2014;33:3639-54.

57. Peters JL, Sutton AJ, Jones DR, et al. Contour-enhanced metaanalysis funnel plots help distinguish publication Bias from other causes of asymmetry. J Clin Epidemiol 2008;61:991-6.

58. Mavridis $\mathrm{D}$, Welton $\mathrm{NJ}$, Sutton $\mathrm{A}$, et al. A selection model for accounting for publication Bias in a full network meta-analysis. Stat Med 2014;33:5399-412.

59. Schwingshackl L, Knüppel S, Schwedhelm C, et al. Perspective: nutrigrade: a scoring system to assess and judge the Meta-Evidence of randomized controlled trials and cohort studies in nutrition research. Advances in Nutrition: An International Review Journal 2016;7:994-1004.

60. Lim SS, Vos T, Flaxman AD, et al. A comparative risk assessment of burden of disease and injury attributable to 67 risk factors and risk factor clusters in 21 regions, 1990-2010: a systematic analysis for the global burden of disease study 2010. Lancet 2012;380:2224-60. 